Marc Gruber / Claudia Venter *

\title{
"Die Kunst, die Zukunft zu erfinden" - Theoretische Erkenntnisse und empirische Befunde zum Einsatz des Corporate Foresight in deutschen Großunternehmen
}

\section{Zusammenfassung}

Studien zur Zukunftsforschung von Unternehmen befassen sich bislang nur einseitig mit Prognoseverfahren, obgleich für die Durchführung der Zukunftsforschung weitere managementspezifische Fragestellungen von Bedeutung sind. Beklagt wird von der Praxis u.a. ein Mangel an Wissen über eine zweckmäßige organisatorische Verankerung der Zukunftsforschung, deren prozessuale Ausgestaltung, den zu wählenden Prognosehorizont und die inhaltliche Ausrichtung der Analysen. Dieser Beitrag wendet sich den offenen Fragen zu und gewährt auf Basis einer qualitativen Untersuchung bei deutschen Großunternehmen erste empirische Einblicke in die weitgehend intransparente Praxis der Zukunftsforschung. Dabei wird der im angloamerikanischen Raum gebräuchliche Terminus "Corporate Foresight" verwendet, um bereits begrifflich den über die Prognoseverfahren hinausgehenden Anspruch zu verdeutlichen. Die Befunde werden unter Einbezug aktueller Erkenntnisse aus der Theorie zum strategischen Management und zum Innovationsmanagement diskutiert. Wie die Ergebnisse zu erkennen geben, schöpfen die Unternehmen den Gestaltungsspielraum und das Potenzial der Zukunftsforschung noch nicht aus. Drei typische Muster des Corporate Foresight deuten sich an. Empfehlungen für die Ausgestaltung des Corporate Foresight runden den Beitrag ab.

JEL-Classification: 0 21, 0 31, 032 .

Keywords: Innovation and Technology Management, Corporate Foresight, Strategic Management. Innovations- und Technologiemanagment; Corporate Foresight; Strategisches Management.

* PD Dr. oec. Marc Gruber, Institut für Innovationsforschung, Technologiemanagement und Entrepre $\neg n e u r s h i p$, Ludwig-Maximilians-Universität München, Kaulbachstr. 45, 80539 München, Tel. 089/2180-5606, Fax: 0892180-6284, email: gruber@bwl.uni-muenchen.de. Dipl.-Kffr. Claudia Venter, BMW Group / EV-43, 80788 München, Tel. 089/382-28202, email: claudia.venter@bmw.de. Die Autoren danken zwei anonymen Gutachtern für hilfreiche Vorschläge zur Überarbeitung des Beitrags.

1 Vgl. Cuhls (2003), S. 94; Lapide (1997), S. 32; v. Reibnitz (1992), S. 9.

2 Vgl. hierzu die Übersicht bei Armstrong (1986), S. 89ff.

3 Vgl. Geschka/Hammer (1997), S. 466f.; Cubls (2003), S. $94 \mathrm{f}$. 


\section{Einführung}

Der Wunsch, die Zukunft vorherzusagen, ist wahrscheinlich so alt wie die Menschheit selbst. Unter allen Versuchen der Zukunftsprognose konnten dabei jene Werke das größte Aufsehen erregen, die den Lauf der gesamten Weltgeschichte prophezeiten, einschließlich ihres vermeintlichen Untergangs. Eine ernst zu nehmende wissenschaftliche Auseinandersetzung mit dem Bereich der Zukunftsprognose lässt sich bis in das 19. Jahrhundert zurückverfolgen ${ }^{4}$.

Die Betriebswirtschaftslehre befasst sich seit den 1960er Jahren intensiver mit der Zukunftsforschung von Unternehmen, wobei sich zunächst vor allem Arbeiten aus der strategischen Unternehmensplanung dieses Bereichs annahmen und einige Methoden für die Langfristprognose vorschlugen ${ }^{5}$. Vor dem Hintergrund komplexer werdender Rahmenbedingungen und immer häufiger eintretender Trendbrüche (Diskontinuitäten) büßten diese vorwiegend auf einfachen Projektionen und Modellrechnungen beruhenden Verfahren jedoch an Nützlichkeit für die Unternehmensplanung ein, so dass in den 80er und 90er Jahren Bandbreiten möglicher Entwicklungen und alternative Entwicklungspfade in die Zukunftsprognose eingebunden wurden ${ }^{6}$.

Obgleich der Praxis heute zahlreiche sophistizierte Verfahren der Zukunftsprognose zur Verfügung stehen, bemängeln jüngere Arbeiten eine noch immer unzureichende Qualität der Prognoseergebnisse und fordern eine Intensivierung der auf Prognoseverfahren ausgerichteten Forschung. ${ }^{7}$ Allerdings wird fast gänzlich übersehen, dass der Nutzen der Zukunftsforschung aus Unternehmenssicht nicht allein von der Güte des gewählten Prognoseverfahrens abhängt, sondern ebenso von weiteren wichtigen Faktoren, die das Management bzw. die Implementierung der Zukunftsforschung im Unternehmen betreffen. Von Bedeutung ist insbesondere die organisatorische Verankerung der Zukunftsforschung im Unternehmen, deren prozessuale Ausgestaltung, die Auswahl des einzubeziehenden Personenkreises, der zu wählende Prognosehorizont und die inhaltliche Ausrichtung der Analysen ${ }^{8}$. In der Literatur finden sich hierzu jedoch kaum Anhaltspunkte.

Der Mangel an theoretischen und empirischen Erkenntnissen zu wichtigen Gestaltungsdimensionen der Zukunftsforschung wird auch von der Praxis beklagt und macht bislang eine unternehmensindividuelle Suche nach geeigneten Lösungen notwendig. Ein Erfahrungsaustausch resp. ein Benchmarking findet aufgrund der hohen strategischen Bedeutung des Bereichs Zukunftsforschung und der Vertraulichkeit entsprechender Informationen i.d.R. nicht statt - ein Umstand der auch Forschungsarbeiten erschwert.

Dieser Beitrag verfolgt das Ziel, erstmalig empirische Einblicke in die verschiedenen Gestaltungsdimensionen der Zukunftsforschung bei deutschen Großunternehmen zu

4 Vgl. Cuhls (2003), S. 94; Lapide (1997), S. 32; v. Reibnitz (1992), S. 9.

5 Vgl. hierzu die Übersicht bei Armstrong (1986), S. 89ff.

6 Vgl. Geschka/Hammer (1997), S. 466f.; Cubls (2003), S. $94 f$.

7 Vgl. z.B. Kahn (2002), S. 134; Lawless (1997), S. 9. Vgl. zum Forschungsstand ebenso Hardie et al. (1998).

8 Ähnlich argumentiert auch Kahn (2002), S. 133f. 
geben und die gewonnenen Einsichten unter Einbindung aktueller Erkenntnisse aus dem Innovationsmanagement und dem strategischen Management zu diskutieren. Dabei wird der im angloamerikanischen Raum gebräuchliche Terminus „Corporate Foresight“ verwendet, um bereits begrifflich den über die reinen Prognoseverfahren (den „Forecasting Techniques“) hinausgehenden Anspruch des Beitrags zu verdeutlichen.

Der Beitrag gliedert sich in sechs Abschnitte. In Abschnitt 2 erfolgt zunächst eine Definition und theoretische Verankerung des Corporate Foresight. Der Stand der Forschung wird in Abschnitt 3 dargelegt. Abschnitt 4 erläutert die Untersuchungsziele und das Design der Studie. Abschnitt 5 berichtet die eigenen empirischen Befunde. Eine Diskussion der Ergebnisse, eine Ableitung von drei Ansätzen des Corporate Foresight und Empfehlungen für die Ausgestaltung des Corporate Foresight runden den Beitrag ab (Abschnitt G).

\section{Definition und theoretische Verankerung des Corporate Foresight}

Das Corporate Foresight ${ }^{9}$ befasst sich im Kern mit der zukunftsorientierten Ausrichtung von Unternehmen und kann daher als Teilbereich des strategischen Innovationsmanagements bzw. allgemeiner des strategischen Managements verstanden werden. In der Literatur wird oftmals auf die frühe Begriffsdefinition von Coates (1985, S. 30) rekurriert, die den prozessualen Charakter des Foresight und die Einbindung in einen größeren Planungsprozess hervorhebt:

„Foresight is the overall process of creating an understanding and appreciation of information generated by looking ahead. Foresight includes qualitative and quantitative means for monitoring clues and indicators of evolving trends and developments and is best and most useful when directly linked to the analysis of policy implications. Foresight prepares us to meet the needs and opportunities of the future."

Dabei bezieht sich das Foresight auf den langfristigen Blick in die Zukunft, der eine aktive Gestaltung zukünftiger Handlungsfelder des Unternehmens ermöglichen soll. Hamel/Prahalad (1994, S. 79) sprechen sogar von einer „Competition for Industry Foresight“, d.h. einem Wettbewerb, der darauf abzielt, die bestmöglichen Annahmen über die Zukunft zu generieren und durch entsprechende Strategien nachhaltigen Einfluss auf die Branchenentwicklung zu nehmen. Das Corporate Foresight ergänzt damit eine meist kurzfristiger orientierte Zukunftsplanung der Unternehmensbereiche und übt für diese eine richtungweisende Funktion aus ${ }^{10}$.

Wie die zitierte Definition darlegt, besteht eine wichtige Aufgabe des Foresight in der Zukunftsprognose (Forecasting), die der systematischen Ermittlung zukünftiger Trends bzw. Diskontinuitäten dient, auf diese Weise zukünftige Chancen und Gefahren identifiziert und die Ideengenerierung im Unternehmen unterstützt. Die Definition bringt

9 In der jüngeren Literatur werden - weitgehend synonym - auch die Begriffe „Strategic Foresight“ und "Strategic Corporate Foresight" verwendet.

10 Vgl. z.B. die empirischen Ergebnisse bei Roberts (1995), S. 51. Siehe auch Armstrong (1986), S. 89. 
jedoch auch zum Ausdruck, dass das Konzept Corporate Foresight deutlich über die reine Anwendung von Prognoseverfahren hinausgeht, indem es im Unternehmen ein allgemeines Verständnis und eine Wertschätzung für Prognosedaten schaffen soll. Angesprochen sind damit Fragen zur Auswahl des an der Zukunftsforschung teilnehmenden Personenkreises (z.B. Einbindung bedeutender Stakeholder), zur organisatorischen Einbettung des Corporate Foresight im Unternehmen und zur Integration der Prognosen in die strategische Planung (z.B. für eine Entwicklung idiosynkratischer Ressourcen) ${ }^{11}$.

In theoretischer Hinsicht leistet das Corporate Foresight einen zentralen, jedoch bislang noch wenig beleuchteten Beitrag zum ressourcenorientierten Ansatz des strategischen Managements, dessen jüngere Forschungsarbeiten auf eine Dynamisierung des Ansatzes („dynamic resource-based view (RBV)“) $)^{12}$ fokussieren. Ausgehend von der Fragestellung, wie die für die Erzielung nachhaltiger Wettbewerbsvorteile erforderliche Heterogenität in der Ressourcenausstattung und dem Fähigkeitenprofil eines Unternehmens zustande kommt, befassen sich Forschungsarbeiten zum dynamischen RBV u.a. mit dem Lebenszyklus von organisationalen Fähigkeiten („capability lifecycles“). ${ }^{13}$ Die Frage, wie Unternehmen identifizieren können, in welchen Bereichen ein Aufbau zukünftig relevanter organisationaler Fähigkeiten förderlich ist, wird dabei oftmals ausgeblendet ${ }^{14}$ oder nur in Teilaspekten bspw. anhand des lerntheoretischen Konzepts eines „local“ bzw. „distant search" thematisiert. Dabei spart letzteres Konzept eine zeitliche Perspektive meist aus, ${ }^{15}$ obgleich u.a. Dierickx/Cool (1989, S. 1504ff.) darauf hinweisen, dass der Aufbau von strategisch wichtigen Fähigkeiten mehrere Jahre dauern kann. Aktivitäten der Zukunftsforschung lassen sich damit als „dynamic capabilities“16 interpretieren, die für die Generierung (fortwährend) neuer Wettbewerbsvorteile erforderlich sind.

\section{Bisherige Erkenntnisse mit Relevanz für das Corporate Foresight}

Im folgenden Überblick zum Stand der Forschung (vgl. auch Tabelle 1) werden zum einen die schon intensiver erforschten Verfahren der Zukunftsprognose behandelt. Zum anderen werden die noch spärlich vorliegenden Erkenntnisse zur inhaltlichen, organisatorischen, prozessualen und personellen Ausgestaltung des Corporate Foresight gewürdigt.

11 Vgl. Cuhls (2003), S. 95ff.; Salo et al. (2003), S. 236.

12 Vgl. Helfat (2000), S. 955ff.; vgl. zu den Grundlagen des ressourcenorientierten Ansatz z.B. Barney (1991), S. 99ff.

13 Vgl. Helfat/Peteraf(2003), S. $997 \mathrm{ff}$.

14 So nennen z.B. Helfat/Peteraf (2003) lediglich Beispiele, die sich auf zeitlich parallel ablaufende Veränderungen im Unternehmensumfeld (u.a. Preisschock) beziehen.

15 Bspw. thematisieren Gavetti/Levinthal (2000) ,search processes that are forward-looking“, rücken jedoch kognitive Fragestellungen in das Zentrum ihres Beitrags. Vgl. ebenso die Kritik bei Levinthal/March (1993), S. 101ff.

16 Siehe hierzu Teece et al. (1997), S. 509ff.; Day (1994b), $43 \mathrm{f}$. 


\section{Tabelle 1: Synopse aktueller Untersuchungen mit Relevanz für das Corporate Foresight}

\begin{tabular}{|c|c|c|c|}
\hline Autoren & Untersuchungsobjekt & $\begin{array}{c}\text { Forschungsansatz } \\
\text { / Stichprobe }\end{array}$ & Befunde \\
\hline $\begin{array}{l}\text { Bonner/ } \\
\text { Ruekert/ } \\
\text { Walker (2002) }\end{array}$ & $\begin{array}{l}\text { Interfunktionale } \\
\text { Neuproduktentwicklungs- } \\
\text { teams in } \\
\text { unterschiedlichen } \\
\text { Branchen }\end{array}$ & $\begin{array}{l}\text { empirisch, } \\
\text { explorativ } \\
(n=95)\end{array}$ & $\begin{array}{l}\text { Steigerung des kreativen Potenzials } \\
\text { von Produktentwicklungsteams } \\
\text { durch: Flexibilität beim Einsatz einer } \\
\text { eigenen Projektkontrolle und eigener } \\
\text { Prozesse/Methoden; Einbindung } \\
\text { in die Gesamtstrategie; selektiver } \\
\text { Eingriff des Top-Managements in die } \\
\text { Prozessaktivitäten }\end{array}$ \\
\hline $\begin{array}{l}\text { Bürgel/Reger/ } \\
\text { Ackel-Zakour } \\
(2002)\end{array}$ & $\begin{array}{l}\text { Technologie- } \\
\text { Früherkennungs- } \\
\text { Prozesse in } \\
\text { internationalen } \\
\text { Unternehmen }\end{array}$ & $\begin{array}{l}\text { empirisch, } \\
\text { explorativ } \\
(\mathrm{n}=21)\end{array}$ & $\begin{array}{l}\text { Erfolgsfaktoren für den TFE- } \\
\text { Prozess: Commitment des Top- } \\
\text { Managements, Prozeßpromotoren, } \\
\text { Interdisziplinarität der Mitarbeiter, } \\
\text { Akzeptanz in der Linienordnung } \\
\text { und in den Geschäftsbereichen, } \\
\text { Motivation, Kommunikation, flexible } \\
\text { Organisationsformen }\end{array}$ \\
\hline $\begin{array}{l}\text { Conway/ } \\
\text { McGuinness } \\
\text { (1986) }\end{array}$ & $\begin{array}{l}\text { Einfluss von } \\
\text { Managementstrukturen } \\
\text { auf die Ideengenerierung } \\
\text { in Branchen mit kurzen } \\
\text { Innovationszyklen }\end{array}$ & $\begin{array}{l}\text { empirisch, } \\
\text { explorativ } \\
(\mathrm{n}=9)\end{array}$ & $\begin{array}{l}\text { Ideengenerierung getrieben durch eine } \\
\text { Vielzahl von Faktoren: kunden- markt- } \\
\text {, wettbewerbs-, technologiegetrieben, } \\
\text { diversifikations- und chancengetrieben; } \\
\text { 3-stufiger Suchprozess: } \\
\text { Problemerkennung, Falsifizierung der } \\
\text { gefundenen Ideen; intensive Aufbereitung; } \\
\text { Kriterien wechseln von Stufe zu Stufe }\end{array}$ \\
\hline $\begin{array}{l}\text { Gausemeier/ } \\
\text { Fink/Schlake } \\
\text { (1998) }\end{array}$ & $\begin{array}{l}\text { Szenariomanagement: } \\
\text { Phasen des } \\
\text { Szenarioprojektes }\end{array}$ & konzeptionell & $\begin{array}{l}\text { Die } 5 \text { Phasen des Szenarioprojektes: } \\
\text { Scenario-Preparation, Scenario- } \\
\text { Analysis, Scenario-Prognostic, Scenario- } \\
\text { Development, Senario-Transfer; die } \\
\text { Heterogenität möglicher Zukunftsbilder } \\
\text { macht Szenarioergebnisse zu einem } \\
\text { wichtigen Hilfsmittel für die strategische } \\
\text { Planung }\end{array}$ \\
\hline $\begin{array}{l}\text { Godet } \\
(2000)\end{array}$ & Szenariomanagement & konzeptionell & $\begin{array}{l}\text { Unterscheidung von zwei Kategorien von } \\
\text { Szenarios: explorativ und antizipatorisch/ } \\
\text { normativ; die Auswahl der verwendeten } \\
\text { Methoden ist v.a. abhängig vom } \\
\text { spezifischen Problem, von Zeit- und } \\
\text { Informationsbarrieren }\end{array}$ \\
\hline $\begin{array}{l}\text { Kahn } \\
(2002)\end{array}$ & $\begin{array}{l}\text { Produkt-Forecasting } \\
\text { zum Kommerzialisierungs- } \\
\text { zeitpunkt }\end{array}$ & $\begin{array}{l}\text { empirisch, } \\
\text { explorativ } \\
(n=168)\end{array}$ & $\begin{array}{l}\text { Starker Einfluss der Marketingabteilung } \\
\text { auf den Forecastingprozess; Präferenz } \\
\text { von qualitativen Forecastingmethoden; } \\
\text { Verwendung mehrerer } \\
\text { Forecastingmethoden während eines } \\
\text { Prozesses; es besteht grundsätzlich } \\
\text { weder ein Zusammenhang der } \\
\text { Vorhersagegenauigkeit mit der Einbindung } \\
\text { einer bestimmten Abteilung noch mit der } \\
\text { Verwendung einer bestimmten } \\
\text { Methode }\end{array}$ \\
\hline
\end{tabular}




\begin{tabular}{|c|l|l|l|}
\hline Autoren & Untersuchungsobjekt & $\begin{array}{l}\text { Forschungsansatz } \\
\text { / Stichprobe }\end{array}$ & \multicolumn{1}{c|}{ Befunde } \\
\hline $\begin{array}{c}\text { Veryzer } \\
(1998)\end{array}$ & $\begin{array}{l}\text { Entwicklungsprozess } \\
\text { radikaler Innovationen }\end{array}$ & $\begin{array}{l}\text { empirisch, } \\
\text { explorativ } \\
(\mathrm{n}=8)\end{array}$ & $\begin{array}{l}\text { Oft kein fomal definierter, fest } \\
\text { vorgeschriebener Szenarionprozess } \\
\text { vorhanden, Konsistenz/Logik des } \\
\text { Prozesses bedeutsam; deutliche } \\
\text { Unterschiede zum Entwicklungsprozess } \\
\text { inkrementeller Innovationen; explorative, } \\
\text { meist technologiegetriebene Prozesse; } \\
\text { Anwesenheit eines Champions wichtig }\end{array}$ \\
\hline $\begin{array}{l}\text { Wilson } \\
(2000)\end{array}$ & $\begin{array}{l}\text { Szenarioprojekte und } \\
\text { ihre Einbindung in die } \\
\text { Unternehmensstrategie }\end{array}$ & konzeptionell & $\begin{array}{l}\text { Eingliederung von Szenarioprojekten/- } \\
\text { ergebnissen in die Strategieplanung des } \\
\text { Unternehmens als entscheidender aber } \\
\text { schwierig umzusetzender Erfolgsfaktor; } \\
\text { Ausweg: Szenarios auf konkrete } \\
\text { Entscheidungsprobleme anwenden }\end{array}$ \\
& & &
\end{tabular}

\subsection{Verfahren der Zukunftsprognose}

Aufgrund einer intensiven, bis in die 60er Jahre zurückreichenden Beschäftigung mit Verfahren der Zukunftsprognose ist nicht weiter erstaunlich, dass in diesem Bereich der Zukunftsforschung die deutlichsten Erkenntnisfortschritte erzielt werden konnten ${ }^{17}$. Der Großteil der Verfahren wurde in den $80 \mathrm{er}$ Jahren entwickelt, ${ }^{18}$ wobei sich das anhaltende Interesse an dieser Thematik bspw. im Rahmen von Coopers (2000) Vorschlag zur Planung radikal neuer Produkte zeigt. In der Literatur hat sich eine Einteilung in quantitative und qualitative Prognoseverfahren durchgesetzt ${ }^{19}$. Veröffentlichungen jüngeren Datums - wie z.B. von Mackay/Metcalfe (2002, S. 221) - empfehlen eine Kombination verschiedener Methoden, um die spezifischen Vor- und Nachteile einzelner Verfahren ausgleichen zu können.

Porter et al. (1991, S. 64) diskutieren die Anwendungsvoraussetzungen und den Nutzen von fünf in der Praxis besonders verbreiteten Prognoseverfahren. Dabei handelt es sich um Aktivitäten des Monitoring, Expertenbefragungen, Szenarioanalysen, Trendextrapolationen und Simulationen. Mit dem Monitoring sollen im Rahmen einer allgemeinen strategischen Frühaufklärung „schwache Signale“ aus dem Unternehmensumfeld geortet werden, aus denen Hinweise auf zukünftige Chancen und Gefahren für Unternehmen gewonnen werden können ${ }^{20}$. Das Monitoring schafft auf diese Weise eine Informationsbasis für die Ideengenerierung und -bewertung im Corporate Foresight ${ }^{21}$. Expertenbe-

17 Siehe z.B. die Arbeiten von Prehoda (1967); Bright (1968); Cetron (1971). Vgl. auch Grupp/Linstone (1999), S. 87.

18 Vgl. Burgelman/Maidique (1988), S. 62.

19 Vgl. Kress/Snyder (1994), S. 195ff.; in der Literatur findet sich zudem eine Einteilung in explorative Methoden, die auf einer Extrapolation von Vergangenheitswerten beruhen, und normativen Methoden, die von einem visionären, zukünftigen Zustand Rückschlüsse auf die Vergangenheit ziehen, vgl. Burgelman/Maidique (1988), S. 66.

20 Siehe auch Ansoff (1976), S. 129ff.; die sich daran anschließende Frage, wie man irrelevante von relevanten frühen Signalen unterscheiden kann, wird im folgenden Abschnitt aufgegriffen. Vgl. zu schwachen Signalen auch Müller-Stewens (1990), Liebl (1996), S. $24 \mathrm{ff}$.

21 Vgl. Porter et al. (1991), S. 64. 
fragungen, wie beispielsweise im Rahmen der Delphianalyse, sind in der Wissenschaft etablierte Verfahren zur Ermittlung qualitativer Aussagen über zukünftige Zustände und eignen sich, wie Studien festgestellt haben, besonders gut für eine langfristige Zukunftsprognose. ${ }^{22}$ Ebenso ist sich die Literatur einig, dass die Szenario-Technik dank ihres breiten Einsatzspektrums geeignete Handlungsempfehlungen und Strategieoptionen im Rahmen der Zukunftsforschung liefern kann ${ }^{23}$. Hingegen ist die Trendextrapolation, die zu den quantitativen Prognoseverfahren zählt, aufgrund ihrer Anwendungsvoraussetzungen von deutlich geringerem Nutzen für die langfristige Prognose. Ähnliche Kritik kann am Verfahren der Simulation geübt werden.

Tabelle 2 gibt vor diesem Hintergrund eine Übersicht zu gängigen Methoden der Zukunftsprognose.

\section{Tabelle 2: Übersicht zu Methoden der Zukunftsprognose24}

\begin{tabular}{|lll|}
\hline Extrapolation & Zielanalysen & Intuitive Methoden \\
\hline Technische Trendanalyse & Implikationsanalyse & Delphianalyse \\
Substitutionsanalyse & Inhaltsanalyse & Nominal Group Conference \\
Growth Limit Analysis & Stakeholderanalyse & Strukturierte/unstrukturierte Interviews \\
Lernkurven & Patentanalyse & Chancenanalyse \\
\hline Musteranalysen & Strat. Frühaufklärung und Planung \\
\hline Analogien & Scanning & \\
Precursor Trends & Monitoring & \\
Morphologie & Szenarioplanung & \\
Feedback-Modelle & Monte Carlo Simulation & \\
\hline
\end{tabular}

\subsection{Inhalt und zeitlicher Horizont der Zukunftsprognosen}

Die Unsicherheit über zukünftige Entwicklungen, die bei langfristigen Prognosen besonders ausgeprägt ist, erfordert es, die Zukunftsforschung inhaltlich auf verschiedenste Prognosefelder auszurichten. Der Informationsbedarf für die strategische Planung bezieht sich dabei sowohl auf das technologische, soziale, ökonomische und ökologische Unternehmensumfeld als auch auf verschiedene institutionelle Akteure wie Mitbewerber, Lieferanten und Kundenx ${ }^{25}$.

Angesichts des relativ umfassenden Informationsbedarfs, einer jedoch begrenzten Kapazität zur Suche und Verarbeitung von Informationen, nehmen sich Forschungsarbeiten im Bereich des strategischen Managements der Frage an, wie Unternehmen bei der

22 Vgl. Burgelman/Maidique (1988), S. 73f.

23 Vgl. v. Reibnitz (1992), S. 14.

24 Nach Vanston (1995).

25 Vgl. Cooper (2000), 1; Geschka/Hammer (1997), S. 465. 
Generierung von Informationen bzw. neuen Wissens vorgehen, welche Arten von schwachen Signalen von besonderer Relevanz für die Erhaltung einer überdurchschnittlichen Wettbewerbsfähigkeit sind und welche kognitiven Modelle von Unternehmern bei der Informationsverarbeitung genutzt werden ${ }^{26}$.

Frühe Forschungsarbeiten wie z.B. von Daniel (1961, S. 111ff.) rücken jene Informationen in den Vordergrund, die sich auf die Erfolgsfaktoren von Unternehmen beziehen und daher die größte Beachtung durch das Management verdienen. Ein solches Vorgehen ist inhaltlich verwandt mit dem vergleichsweise jüngeren Konzept des „local search“, das aussagt, dass Unternehmen nach neuen Informationen und innovativen Lösungen vor allem in der Nachbarschaft ihrer bisherigen Expertise bzw. Wissensbasis suchen ${ }^{27}$. Cohen/ Levinthal (1990, S. 128ff.) verwenden den Begriff „absorptive capacity“, um zu verdeutlichen, dass die Fähigkeiten eines Unternehmens zur Assimilierung und Integration von neuem technologischen Wissen eng mit den bisherigen Aktivitäten in der F\&E und dem schon im Unternehmen vorhandenen Wissen verbunden sind. Stuart/Podolny (1996, S. $21 \mathrm{ff}$.) belegen dies empirisch bei Halbleiterunternehmen, deren Patentierungsaktivitäten vor allem auf jene Technologiebereiche fokussiert sind, in denen die Unternehmen bereits früher Patente angemeldet haben ${ }^{28}$. In der Folge akkumulieren diese Unternehmen eine besondere Expertise in ihrem Bereich, laufen dabei allerdings auch Gefahr gewissen „core rigidities“ (Leonard-Barton, 1992), „competency traps“ (Levitt/March, 1993) und einer „escalation of commitment“ (Staw/Ross, 1987) zu erliegen. Jüngere Forschungsarbeiten betonen daher, dass die langfristige Erhaltung von Wettbewerbsvorteilen vor allem auf der Fähigkeit beruht, als Unternehmen einen „distant search“ durchführen und die vorhandene Wissensbasis markant erweitern zu können ${ }^{29}$. Angesichts des relativ langen Prognosezeitraums dürfte es den am Foresight-Prozess beteiligten Personen tendenziell auch leichter fallen, sich gedanklich von den gegenwärtigen Unternehmensaktivitäten zu lösen ${ }^{30}$, vermeintliche „core rigidities“ zu überwinden und strategische Optionen der Unternehmensentwicklung freier zu assoziieren. Vom Unternehmen können auf dieser Basis verschiedene Realoptionen für alternative Entwicklungspfade aufgebaut werden, die bei abnehmender Unsicherheit über die Entwicklung entweder intensiver verfolgt oder verworfen werden können ${ }^{31}$. Aus lerntheoretischer Sicht weisen Levinthal/March (1993) jedoch darauf hin, dass Personen weiter in der Zukunft liegende Entwicklungen häufig vernachlässigen.

Über die Länge einer „langfristigen“ Prognose gibt es in der Literatur unterschiedliche Auffassungen. Manche Autoren wie Hogarth/Makridakis (1981, S. 122) sprechen bereits ab einem Prognosezeitraum von zwei Jahren von „long-range forecasting“. Für die Mehrzahl der Forscher bedeutet eine langfristige Zukunftsprognose, dass ein Zeitraum von mindestens

26 Vgl. auch Day/Schoemaker (2000), S. 19ff., Gavetti/Levinthal (2000), S. 113ff.; Day/Nedungadi (1994), S. $31 \mathrm{ff.}$

27 Vgl. Stuart/Podolny (1996), S. 21ff. Vgl. in diesem Kontext ebenso Day (1994a), $12 \mathrm{f}$.

28 Entsprechend weisen Martin/Mitchell (1998, S. 753ff.) darauf hin, dass Aktivitäten des „local search“ dazu führen, dass bestehende Unternehmen Produktdesigns einführen, die ähnlich ihrer bereits bestehenden Produkte sind.

29 Vgl. RosenkopflNerkar (2001), S. 288; Sorenson/Stuart (2000), S. 81ff.; March (1994), S. 71ff.; Gavetti/Levinthal (2000), S. $113 \mathrm{ff}$.

30 Vgl. auch die analogen Ausführungen bei Day/Schoemaker (2000), S. 19.

31 Vgl. Day/Schoemaker (2000), S. 26, McGrath (1997), 13ff.; Trigeorgis (1999). 
fünf bis zehn Jahren betrachtet wird, wobei der jeweilige Branchenkontext und insbesondere die Länge des Produkt- oder Technologielebenszyklus zu berücksichtigen sind ${ }^{32}$.

\subsection{Organisatorische Verankerung und prozessuale Ausgestaltung des Corporate Foresight}

Aus der Literatur lassen sich verschiedene Optionen für die organisatorische Verankerung des Corporate Foresight im Unternehmen ableiten. Die konkrete Ausgestaltung wird dabei u.a. von der generellen Bedeutung, die dem Corporate Foresight vom Management beigemessen wird und von den bereits existierenden Strukturen (z.B. eigene F\&E-Abteilung) beeinflusst ${ }^{33}$. Die Studie von Bürgel et al. (2002, S. 25ff.) zeigt, dass Aktivitäten der Technologie-Früherkennung auf Konzernebene, Geschäftsbereichsebene und in virtuellen Strukturen durchgeführt werden können ${ }^{34}$. Roberts (1995, S. 52) und Kappel (2001, S. 39) verweisen auf die Gefahr, dass Bereichsabteilungen aufgrund ihrer größeren Nähe zum Produkt und zum Kunden kurzfristige gegenüber langfristigen Entwicklungsprojekten vorziehen und somit im Konzern die Bildung neuer Kernkompetenzen ggf. vernachlässigt wird.

Untersuchungsergebnisse aus den Vereinigten Staaten lassen einen deutlichen Trend zur Institutionalisierung der Zukunftsforschung erkennen ${ }^{35}$. Während diese Aktivitäten bis in die 80er Jahre durch Mitarbeiter der F\&E-Abteilung bzw. einer Stabstelle „Strategieentwicklung" ausgeübt wurden, zeichnet sich seit Mitte der 90er Jahre eine Professionalisierung der Zukunftsforschung ab, die sich in der Bildung eigener Abteilungen bzw. Stabstellen sowie der Schaffung von Vollzeitstellen niederschlägt ${ }^{36}$.

Korrespondierend zur organisatorischen Verankerung des Corporate Foresight kann auch der Foresight-Prozess entweder als zeitlich begrenzte oder als Daueraufgabe verstanden werden. Forschungsergebnisse weisen in diesem Zusammenhang besonders auf die Vorteile eines kontinuierlichen Prozesses hin, der fest in der Unternehmensorganisation eingebunden und auf die übergeordneten strategischen Ziele und Erfolgsgrößen des Gesamtunternehmens ausgerichtet ist ${ }^{37}$.

\subsection{Personelle Ausgestaltung des Corporate Foresight}

Mit Blick auf die personelle Ausgestaltung des Corporate Foresight lassen sich erste Hinweise der Literatur zum Innovationsmanagement entnehmen. Grundlegend ist die

32 Vgl. Bürgel et al. (2002), S. 24; Burgelman/Maidique (1988), S. $59 \mathrm{f}$.

33 Vgl. Elikai et al. (1999), S. 16.

34 Dies belegen auch empirische Ergebnisse von Kahn (2002, S. 135) zu Forecasting-Aktivitäten der Neuproduktentwicklung in der Marketing-/Vertriebsabteilung.

35 Vgl. Jain (1999), S. 2; Jain (2002a), S. 2.

36 Vgl. auch Burgelman/Maidique (1988), S. 79; Roberts (1995), S. 44ff. Dabei hat sich die Zukunftsforschung vor allem bei jenen Unternehmen relativ früh als eigene Funktion verbreitet, die in Branchen mit vergleichsweise langen Zyklen tätig sind, wie z.B. in der Pharma- oder Mineralölbranche. Vgl. Jain (2002a), S. 3.

37 Vgl. Jain (1999), S. 28. 
Einsicht, dass Personen mit unterschiedlichem Hintergrund (Ausbildung, Abteilungszugehörigkeit) eingebunden werden sollen, um zu vermeiden, dass Prognosen durch spezifische „Weltsichten“ geprägt werden und einseitig ausfallen ${ }^{38}$. Dies bestätigt auch die Gruppenforschung ${ }^{39}$. Gleichfalls lassen empirische Ergebnisse den Schluss zu, dass sich eine heterogene Zusammenstellung der am Foresight-Prozess beteiligten Personengruppen und insbesondere die frühe Integration von späteren Umsetzern und Entscheidern (Machtpromotoren) positiv auf die Akzeptanz und Realisierung der Zukunftsprognosen auswirken ${ }^{40}$. Cuhls (2003, S. 96) wertet diese Beteiligung verschiedener Personen aus dem Innovationssystem aufgrund der damit verbundenen Mobilisierungs- und Kommunikationseffekte als gleich wichtig für das Foresight wie die eigentlichen Prognoseergebnisse. Offen ist, welches Gewicht Vertretern einzelner Abteilungen (insb. F\&E und Marketing) jeweils zukommen soll.

\section{Ziele und Design der empirischen Fallstudienuntersuchung}

\subsection{Forschungsziele und -fragen}

Obige Ausführungen zum Stand der Forschung machen deutlich, dass sowohl ein inhaltliches Forschungsdefizit als auch ein Mangel an empirisch fundierten Erkenntnissen im Bereich der Zukunftsforschung von Unternehmen existiert. Es liegt kaum Wissen über die Realisierung des Corporate Foresight in Unternehmen vor, das einerseits die Forschung befruchten und andererseits als Basis für ein Benchmarking in der Praxis dienen könnte ${ }^{41}$. Vorliegende Untersuchung verfolgt daher das Ziel, die theoretisch diskutierten Gestaltungsdimensionen der Zukunftsforschung einer empirischen Exploration zu unterziehen. Dazu wurde eine vergleichende Fallstudienanalyse bei deutschen Großunternehmen durchgeführt.

Wegleitend für die Durchführung der Untersuchung waren die folgenden drei Forschungsfragen:

(1) Wie ist das Corporate Foresight in deutschen Großunternehmen ausgestaltet (eingesetzte Prognoseverfahren, Inhalte, Prognosehorizont, organisatorische Verankerung, prozessuale Ausgestaltung, personelle Besetzung?

Diese explorative Fragestellung gibt einerseits einen ersten Aufschluss über den Professionalisierungsgrad der Zukunftsforschung in deutschen Großunternehmen, andererseits gewährt sie erstmals empirische Einblicke in die konkrete Realisierung zentraler Gestaltungsaspekte der Zukunftsforschung.

39 Vgl. bspw. Williams/O'Reilly (1998), S. $77 \mathrm{ff}$.

40 Vgl. Bürgel et al. (2002), S. 40; Wilson (2000), S. 24.

41 Die befragten Experten haben in diesem Zusammenhang darauf hingewiesen, dass sie nur ansatzweise Kenntnis über Foresight-Prozesse anderer Unternehmen haben und ein Benchmarking zur Verbesserung der eigenen Prozesse deshalb bisher nicht möglich ist. 
(2) Unterscheiden sich die Unternehmen bei der Ausgestaltung ihrer Zukunftsforschung? Lassen sich bestimmte Muster/Ansätze bei der Ausgestaltung erkennen?

Aus bisherigen Forschungsarbeiten ist nicht ersichtlich, inwiefern es Unterschiede bei der Ausgestaltung der Zukunftsforschung einzelner Unternehmen gibt und welche Gründe die möglicherweise vorhandene Heterogenität haben könnte. Die vorhandene Literatur lässt bislang lediglich vermuten, dass der jeweilige Branchenkontext die Länge des Prognosehorizonts maßgeblich beeinflusst ${ }^{42}$.

(3) Welche Erfahrungen haben die Unternehmen mit dem Corporate Foresight gesammelt und welche Ansatzpunkte existieren, um die Zukunftsforschung weiter zu verbessern?

Bislang findet ein Erfahrungsaustausch zur Zukunftsforschung aufgrund der hohen strategischen Bedeutung und der Vertraulichkeit entsprechender Informationen nicht statt. Zur Verbesserung ihres Corporate Foresight sind Unternehmen daher auf eigene Erfahrungen und auf verfügbare wissenschaftliche Erkenntnisse angewiesen.

\subsection{Design der Untersuchung und methodisches Vorgehen}

Wie sich bereits bei der Anbahnung des Forschungsprojekts deutlich gezeigt hat, ist die Bereitschaft von Unternehmen, Auskunft zu ihren Aktivitäten der Zukunftsforschung zu geben, äußerst gering. Zwar war eine gewisse Zurückhaltung bei der Preisgabe von Informationen zu diesen strategisch bedeutsamen Aktivitäten zu erwarten gewesen, dennoch war die (zunächst) nur geringe Auskunftsbereitschaft - insbesondere im Vergleich $\mathrm{zu}$ anderen strategischen Fragestellungen in der Betriebswirtschaftslehre - überraschend ${ }^{43}$. Um einen empirischen Zugang zu dieser Thematik zu erhalten, war es vor diesem Hintergrund am viel versprechendsten, die erforderlichen Informationen zur Zukunftsforschung fallstudienbasiert zu erheben. Insbesondere konnten hierdurch umfassende vertrauensbildende Maßnahmen ergriffen werden.

Die Wahl eines fallstudienbasierten, auf qualitativen ${ }^{44}$ Forschungsmethoden beruhenden Ansatzes hat sich bereits in einer Reihe bisheriger Studien in diesem Bereich bewährt ${ }^{45}$, da ein solches Vorgehen eine gründliche Auseinandersetzung mit dem Untersuchungsobjekt gestattet. Gemäß Eisenhardt (1989) eröffnet insbesondere eine vergleichende Analyse von Fallstudien ein großes Potenzial zur Erkenntnisgewinnung. Die Vergleichbarkeit von Ergebnissen zählt zu den Hauptprinzipien einer fundierten Theoriebildung im Sinne des „Grounded Theory Approach“ ${ }^{46}$.

42 Vgl. Burgelman/Maidique (1988), S. 59f.; Bürgel et al. (2002), S. 24.

43 Dies begründet zum Teil auch den Mangel an empirischen Studien zur Zukunftsforschung von Unternehmen.

44 Vgl. zu den allgemeinen Merkmalen und Gütekriterien qualitativer Forschung Mayring (1990), S. 9ff. und 103ff.; vgl. ebenso Lamnek (1995), S. $93 \mathrm{ff}$.

45 Vgl. z.B. die Untersuchung von Veryzer (1998); Folkerts/Hauschildt (2002).

46 Vgl. Eisenhardt (1989), S. 532ff.; vgl. zudem grundlegenden Ausführungen von Dougherty (2002, S. 849ff.), Lamnek (1995, S. 114), Glaser/Strauss (1967) sowie Strauss/Corbin (1994) zu den Forschungsmethoden des „Grounded theory building research“. 
Im Rahmen der vorliegenden Studie wurden deshalb im gleichen Zeitraum insgesamt acht Fallstudien zur Zukunftsforschung von deutschen Großunternehmen erhoben. Die acht Unternehmen wurden auf Basis einer typischen Auswahl ${ }^{47}$ für die Untersuchung gewonnen, wobei es sich um Unternehmen aus der Elektroindustrie, chemischen Industrie, Automobilindustrie, Halbleiterindustrie und der Telekommunikationsbranche handelt. Die Vergleichbarkeit der erhobenen Informationen wurde dadurch zu gewährleisten versucht, dass es sich um Unternehmen ähnlicher Größenordnung handelt, die mit einer Ausnahme ihren Hauptsitz in Deutschland haben und weltweit agieren. Der durchschnittliche Umsatz der Fallstudienunternehmen betrug im Geschäftsjahr 2003 rund 70 Mrd. Euro. Im Mittel wiesen sie eine Mitarbeiterzahl von rund 200.000 Beschäftigten aus ${ }^{48}$.

Dem Prinzip der Triangulation folgend wurde zur Datenerhebung eine Kombination von Methoden gewählt, so dass Quervergleiche von Daten unterschiedlicher Quellen und damit eine Steigerung der Validität möglich wurde ${ }^{49}$. Als hauptsächliche Datenquellen dienten Dokumentenanalysen und Expertengespräche (vgl. Abbildung 3). Als Interviewpartner standen in den einzelnen Unternehmen in nahezu allen Fällen mehrere Personen (z.B. Projekt- bzw. Abteilungsleiter aus dem Innovationsmanagement oder einem anderen mit dem Corporate Foresight betrauten Bereich) zur Verfügung, so dass einem für die Innovationsforschung nachgewiesenen ,informant bias" ${ }^{\circ 0}$ entgegengewirkt werden konnte. Die Befragung erfolgte anhand eines halbstandardisierten, pre-getesteten Fragebogens, der auf Basis der einschlägigen Literatur und anhand mehrerer Diskussionen mit Experten aus der Praxis erarbeitet wurde. Der Fragebogen umfasste sechzehn offene Hauptfragen mit jeweiligen Unterfragen zu den schon angesprochenen Gestaltungsaspekten der Zukunftsforschung. Den Interviewpartnern wurde vorab der Fragebogen zu Verfügung gestellt, um ihnen die Möglichkeit zu geben, die für die Beantwortung der Fragen erforderlichen Informationen zu beschaffen. Die Expertengespräche dauerten durchschnittlich zwei Stunden und wurden vom Interviewer protokolliert. Anschließend wurden die Protokolle den Befragten zur Überprüfung der erhobenen Informationen zugesandt.

\section{Tabelle 3: Vorgehensweise der qualitativen Untersuchung}

\begin{tabular}{|l|l|}
\hline Quelle & Vorgehensweise \\
\hline Literaturanalyse & $\begin{array}{l}\text { Analyse der wissenschaftlichen Literatur mit Fokus auf Prognoseverfahren, } \\
\text { Innovationsmanagement, strategisches Management und } \\
\text { Zukunftsforschung }\end{array}$ \\
\hline Gruppendiskussion 1 & Moderierte Diskussion von Corporate-Foresight-Experten \\
\hline- & Identifizierung offener Forschungsfragen und Fragebogenerstellung \\
\hline
\end{tabular}

47 Vgl. zur Auswahl typischer Fälle Stier (1999), S. 118.

48 Die Hälfte der Unternehmen in der Untersuchung beschäftigte im Jahr 2003 zwischen 100.000 und 200.000 Mitarbeitern und erzielte einen jährlichen Umsatz zwischen 30 und $40 \mathrm{Mrd}$. €. Drei Unternehmen wiesen eine Mitarbeiterzahl von über 300.000 Mitarbeitern und einen Umsatz von über $100 \mathrm{Mrd}$ € aus, ein Unternehmen beschäftigte weniger als 60.000 Mitarbeiter und erzielte einen Umsatz von weniger als $20 \mathrm{Mrd}$. (Geschäftsberichte 2003).

49 Vgl. hierzu auch Yin (1994), S. 14.

50 Vgl. hierzu Ernst (2003), S. $1249 \mathrm{ff}$. 


\begin{tabular}{|l|l|}
\hline Quelle & Vorgehensweise \\
\hline Experteninterviews 1 & Pre-test des Fragebogens und punktuelle Überarbeitung des Instruments \\
\hline Experteninterviews 2 & $\begin{array}{l}\text { Halbstandardisierte Leitfadeninterviews mit 15 Experten aus insgesamt } \\
\text { 8 Unternehmen; durchschnittliche Gesprächsdauer 2 Stunden; } \\
\text { Protokollierung }\end{array}$ \\
\hline Dokumentenanalyse & $\begin{array}{l}\text { Umfassende Analyse von internen Unternehmensunterlagen zum } \\
\text { Themen-bereich Corporate Foresight }\end{array}$ \\
\hline Experten & Prüfung der Interviewprotokolle \\
\hline- & Fallstudienaufbereitung und -analyse \\
\hline Gruppendiskussion 2 & $\begin{array}{l}\text { Moderierte Diskussion der Resultate mit Corporate-Foresight-Experten } \\
\text { und Validierung der Ergebnisse }\end{array}$ \\
\hline
\end{tabular}

\section{Empirische Befunde}

Die Vertreter der Fallstudienunternehmen hatten erhebliche Bedenken, sich zu den Aktivitäten der Zukunftsforschung zu äußern. Um den Zugang zu den erforderlichen Informationen zu erhalten und deren Vertraulichkeit zu wahren, musste den beteiligten Unternehmen eine anonymisierte Darstellung der Ergebnisse zugesichert werden. Nachfolgend sind die Fallstudienunternehmen daher mit den Buchstaben A bis $\mathrm{H}$ bezeichnet. Erkennbar ist allerdings die jeweilige Branchenzugehörigkeit.

\subsection{Verwendete Verfahren der Zukunftsprognose}

Wie oben dargelegt wurde, stehen Unternehmen zahlreiche Prognoseverfahren zur Verfügung. Die Fallstudien bestätigen, dass sich zur Zukunftsprognose besonders eine kombinierte Anwendung quantitativer und qualitativer Verfahren empfiehlt, wobei in erster Linie qualitative Verfahren verwendet werden, um Langfristprognosen zu erstellen. Unerwartet ist der Befund, dass von den untersuchten Unternehmen ein nahezu identisches Set an Prognoseverfahren eingesetzt wird, das im Kern das Monitoring und Scanning, die Delphianalyse und die Szenarioanalyse umfasst.

Kontinuierlich stattfindende Aktivitäten des Monitoring und des Scanning werden von allen Unternehmen als grundlegend für die mittel- bis langfristige Zukunftsforschung erachtet. Dabei setzen nahezu alle Unternehmen Trendscouts ein, die in weltweit führenden Märkten bzw. technologischen Zentren („Hot Spots“) das aktuelle Umfeld und sich abzeichnende Entwicklungen beobachten. Zur Ermittlung von schwachen Signalen werden von den Trendscouts ebenso Medienanalysen und Expertengespräche mit Forschungseinrichtungen durchgeführt. Ergänzt werden diese Aktivitäten durch spezifische Trendanalysen von Vertretern einzelner Unternehmensbereiche.

Intensiv genutzt wird auch die Delphianalyse, wobei hier einschränkend anzumerken ist, dass fast alle Unternehmen aufgrund der hohen Zeitbeanspruchung und der vergleichsweise hohen Kosten dieser Art der Expertenbefragung auf eine vereinfachte Variante zurückgreifen. Beispielsweise werden einzelne Iterationsschritte verkürzt und Experten nur einmalig befragt. 
Wie die Fallstudien zeigen, wird die Szenarioanalyse als das wichtigste Verfahren der mittel- bis langfristigen Zukunftsprognose angesehen. Die Unternehmen wählen allesamt eine Vorgehensweise, die kaum von den in der Literatur dargestellten Schritten - insbesondere des von v. Reibnitz (1992) empfohlenen Vorgehens - abweicht und auf einer Extrapolation der Gegenwart in die Zukunft beruht. Die Bewertung und Auswahl der für die Szenarioerstellung als relevant erachteten Trends erfolgt dabei weniger anhand harter Kriterien, als vielmehr auf Basis einer „gefühlsmäßigen“ Beurteilung durch die am Prozess beteiligten Personen. Intensiv genutzt werden die von den Trendscouts ermittelten Informationen. Bei der Generierung der Szenarien stehen Kriterien wie Plausibilität und Konsistenz im Vordergrund, wobei nur bei einem der betrachteten Fälle auch scheinbar unmögliche Szenarien gebildet werden, mit dem Ziel, durch Loslösung von bewährten Denkstrukturen die Kreativität zu fördern und neue strategische Optionen zu entdecken. Bezüglich der Anzahl der zu bildenden Szenarien gab der Großteil der Unternehmen an, zwei bis drei plausible Alternativszenarien zu generieren, wobei ein Unternehmen neben einem konsistenten Trendszenario auch stets zwei Extremszenarien erarbeitet, um damit ein möglichst breites Spektrum potenzieller Entwicklungen abzudecken.

Bemerkenswert ist zudem, dass zwei Fallstudienunternehmen die im Rahmen der Szenarioanalyse durchgeführte Extrapolation durch eine Retropolation ergänzen. Während die Extrapolation den Vorteil aufweist, auf eine bekannte Ausgangsbasis für die Zukunftsbetrachtung zurückgreifen zu können, ist mit ihrer Anwendung auch die Gefahr verbunden, dass sich Diskontinuitäten kaum prognostizieren lassen. Diesem Manko versucht die Retropolation zu begegnen, indem für einen gewählten langfristigen Zeithorizont ein „Bild der Zukunft“ entworfen wird, das einem ganzheitlichen Ansatz folgend möglichst viele Einflussfaktoren berücksichtigt und anschließend auf die Gegenwart retropoliert wird. Durch die Zusammenführung von Extrapolation und Retropolation in konsistente Szenarien können Aufgabenfelder und spezifische Problemstellungen identifiziert werden, die in der Gegenwart als erste robuste Schritte angegangen werden müssen, um langfristig wettbewerbsfähig zu bleiben ${ }^{51}$.

Für die Bewertung der aus den Szenarien stammenden produkt-, technologie- bzw. marktbezogenen Ideen werden verschiedene Kriterien herangezogen. Ein für alle Befragten übergeordnetes Kriterium ist der „Fit“ mit der langfristigen Unternehmensstrategie, wodurch u.a. die Breite der künftigen unternehmerischen Aktivitäten definiert wird. Zur Bewertung werden auch klassische Markt- oder Technologiekennzahlen wie die mögliche Marktgröße, das Marktwachstum, die Reife der Technologie und deren Anwendungsbreite herangezogen, wobei Einigkeit darin besteht, dass die ermittelten Werte aufgrund der hohen Unsicherheit von Langfristinnovationen nur vage Aussagen liefern können. Der Grad der Unsicherheit wird häufig als zusätzliches Kriterium zur Priorisierung von Szenarien bzw. von Innovationsideen verwendet.

Ziel des abschließenden Schritts einer Szenarioanalyse ist, die erarbeiteten Erkenntnisse in einer Leitstrategie zusammenzufassen und für die Umsetzung im Unternehmen vorzubereiten. Bei fast allen Unternehmen endet die Szenarioanalyse mit einer Präsenta-

51 Vgl. hierzu auch die Ausführungen von Weyrich (2002), S. 73; Gruber et al. (2003), S. $285 f f$. 
tion der Ergebnisse und einer Ableitung von Handlungsempfehlungen für den Konzern und einzelne Geschäftsbereiche. Eine regelmäßige Einarbeitung der Resultate in die Unternehmensstrategie erfolgt jedoch nur bei drei Unternehmen, eine Umsetzung in der F\&E sogar nur bei zwei Unternehmen. Einen besonderen Fall stellt der Automobilkonzern D dar, bei dem die Resultate lediglich Innovationsimpulse für nachfolgende Bereiche liefern sollen, jedoch keine festen strategischen Vorgaben.

Obgleich sich also die Unternehmen in der Wahl der verwendeten Prognoseverfahren sehr ähnlich sind, unterscheiden sie sich erheblich in der Nutzung der Prognoseergebnisse (vgl. Abbildung 4).

\section{Tabelle 4: Nutzung der Prognoseergebnisse}

\begin{tabular}{|c|c|c|c|c|c|c|c|c|}
\hline & \multicolumn{3}{|c|}{ Elektroindustrie } & \multicolumn{2}{|c|}{ Automobilindustrie } & \multirow{2}{*}{$\begin{array}{c}\text { Chemie } \\
\text { F }\end{array}$} & \multirow{2}{*}{$\begin{array}{c}\text { Telekom. } \\
\text { G }\end{array}$} & \multirow{2}{*}{$\begin{array}{c}\text { Halbleiter } \\
\text { H }\end{array}$} \\
\hline & A & B & C & D & $\mathbf{E}$ & & & \\
\hline $\begin{array}{l}\text { Aufzeigen von } \\
\text { Trends/Szenarien }\end{array}$ & $\bullet$ & $\bullet$ & $\bullet$ & $\bullet$ & $\bullet$ & $\bullet$ & $\bullet$ & $\bullet$ \\
\hline $\begin{array}{l}\text { Ableitung von } \\
\text { Handlungs- } \\
\text { empfehlungen }\end{array}$ & $\bullet$ & - & $\bullet$ & & $\bullet$ & $\bullet$ & $\bullet$ & - \\
\hline $\begin{array}{l}\text { Regelmäßige } \\
\text { Einarbeitung in } \\
\text { Unternehmens- } \\
\text { strategie }\end{array}$ & & & • & & & $\bullet$ & & $\bullet$ \\
\hline $\begin{array}{l}\text { Umsetzung in } \\
\text { der F\&E }\end{array}$ & & & & & • & • & & \\
\hline
\end{tabular}

\subsection{Inhalt und zeitlicher Horizont der Zukunftsprognosen}

Angesichts der zum Teil unterschiedlichen Branchenzugehörigkeit der Unternehmen mag es zunächst nicht weiter verwundern, dass sich auch die Inhalte und die Zeithorizonte ihrer Prognosen unterscheiden. Ein Vergleich der Fallstudien zeigt allerdings den überraschenden Befund, dass selbst bei Unternehmen einer Branche sehr deutliche Unterschiede in diesen Gestaltungsaspekten bestehen.

Bezüglich der gewählten Prognosehorizonte weisen die Unternehmen eine relativ große Schwankungsbreite von 5 bis zu 25 Jahren auf, wobei sich der maximale Zeithorizont der Prognosen beim Großteil der Unternehmen auf 15 bis 20 Jahre beläuft. Jedoch kann die unterschiedliche Länge des Prognosezeitraums, wie die bestehende Literatur zur Zukunftsforschung größtenteils suggeriert, nicht alleine auf die Branchenzugehörigkeit zurückgeführt werden (vgl. Abbildung 5). Vielmehr müssen zur Erklärung des Prognosezeitraums neben branchenspezifischen auch firmenspezifische Aspekte - wie z.B. der vom Management postulierte Bedarf an Langfristprognosen - berücksichtigt werden. So befasst sich Unternehmen B (Elektroindustrie) in seiner Zukunftsforschung mit produkt- und 
marktnahen Fragestellungen und nicht mit Themen der reinen Grundlagenforschung. Den Prognosen liegt daher auch ein Betrachtungszeitraum von lediglich zwei bis fünf Jahren zugrunde, mit dem Ziel, konkrete Vorgaben für Entwicklungsprojekte abzuleiten. Demgegenüber erstellt Unternehmen A, das ebenso aus der Elektroindustrie stammt, mit seinen Zukunftsprognosen ein ganzheitliches Bild der Zukunft, das erst in weiterführenden Projekten zur Generierung von Produktideen genutzt wird.

Interessant ist auch der Vergleich der beiden Automobilkonzerne D und E, deren Prognosen besonders langfristig ausgerichtet sind. Beide Unternehmen nutzen ihre Zukunftsforschung um allgemeine Visionen für die Unternehmensentwicklung aufzustellen und daraus unternehmensweite Strategien abzuleiten, die zur Entscheidungsunterstützung auf verschiedenen Führungsebenen herangezogen werden. Unternehmen D hat zudem spezifische Innovationsfelder und dazugehörige „Top-Themen“ vordefiniert, die einer jährlichen Überprüfung unterliegen und im Fall neu zu erschließender Potenziale konkrete Innovationsprojekte anstoßen. Unternehmen $\mathrm{E}$ verzichtet hingegen auf vordefinierte Untersuchungsbereiche, sondern nutzt globalere Analysen um Produktideen auf Basis übergreifender Trends zu entwerfen und zu bewerten. Gleichfalls werden von einzelnen Geschäftsbereichen Prognoseaufträge an die Zukunftsforschung vergeben.

\section{Abbildung 1: Prognosehorizont und Inhalte des Corporate Foresight}

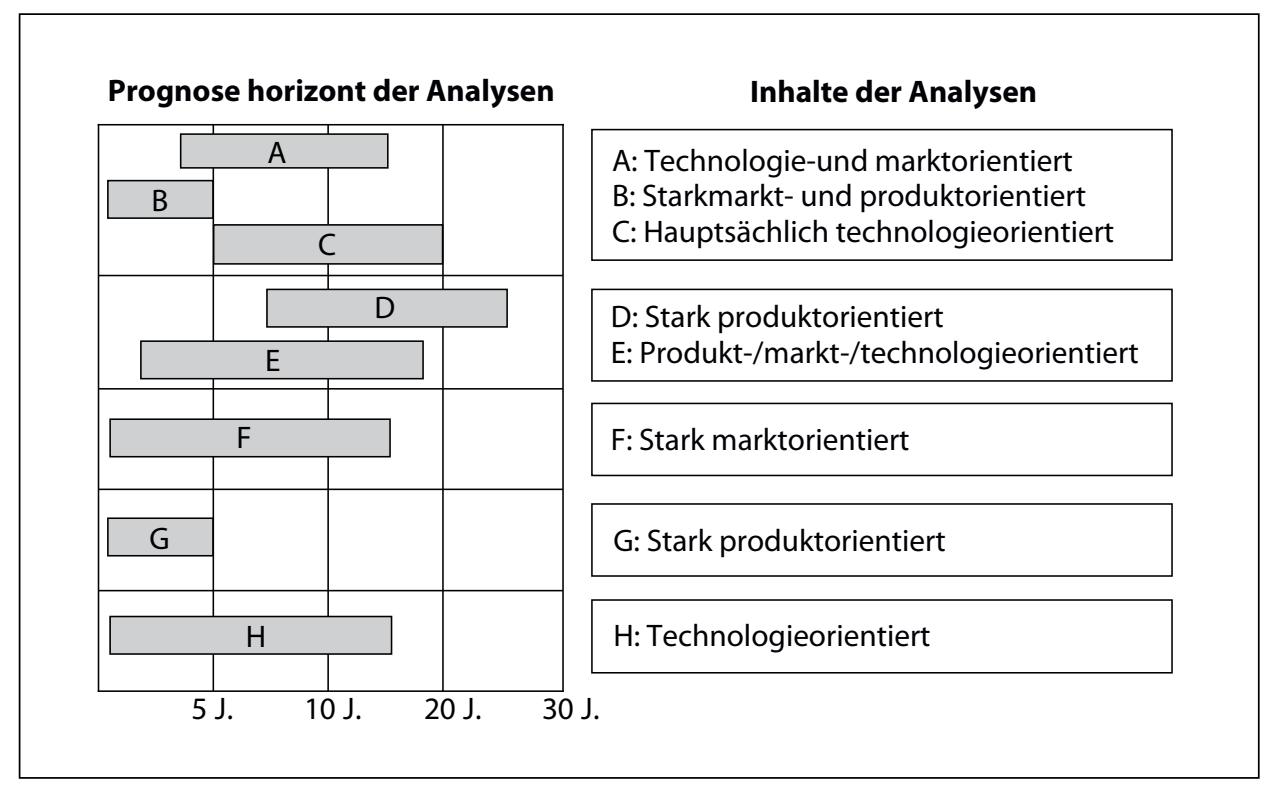

Stellt man die inhaltliche Ausgestaltung der Zukunftsforschung bei allen Unternehmen gegenüber (vgl. Abbildung 1), so sticht neben den Unterschieden im Prognosehorizont auch die heterogene inhaltliche Schwerpunktsetzung der Prognosen ins Auge. Diese ist von besonderem Interesse, weil die Inhalte der Zukunftsforschung als Informationen in 
die strategische Planung einfließen und damit Rückschlüsse auf das jeweilige Strategieverständnis der Unternehmen zulassen. Insbesondere gewähren sie Einblick in die Frage, welche Bedeutung technologiebezogenen, marktbezogenen und produktbezogenen Informationen im Rahmen der strategischen Planung zugeschrieben wird. Wie die Abbildung zeigt, unterscheiden sich die Unternehmen hier relativ deutlich voneinander, wobei nur ein Unternehmen technologie-, markt- und produktbezogene Informationen als gleich bedeutend für seine Analysen erachtet.

Ebenso können Zusammenhänge zwischen den jeweiligen inhaltlichen Schwerpunkten und dem jeweils gewählten Prognosehorizont festgestellt werden. So lässt sich erkennen, dass jene Unternehmen, die sich in ihrer Zukunftsforschung vor allem mit der künftigen Veränderung von Technologien befassen, einen tendenziell längeren Prognosehorizont als relevant erachten, als Unternehmen, die einen Produkt- und/oder Marktfokus verfolgen. Eine mögliche Erklärung hierfür kann der ressourcenorientierte Ansatz liefern. ${ }^{52}$ Eine seiner Grundaussagen ist, dass der Lebenszyklus von Kernkompetenzen i.d.R. länger andauert als der Lebenszyklus von Produkten. Insofern lässt sich nachvollziehen, dass sich jene Unternehmen, die sich mit der zukünftigen Entwicklung von Technologien (im Sinne von Kernkompetenzen) befassen, auch langfristiger in ihren Zukunftsprognosen orientieren als Unternehmen, die sich mit der künftigen Entwicklung von Produkten auseinandersetzen. Mit Blick auf die grundsätzliche Zielsetzung des Corporate Foresight, die organisationale Evolutionsplanung zu unterstützen, ist eine rein produktorientierte Ausrichtung damit kritisch zu hinterfragen.

Die Literatur zum strategischen Management weist ebenso darauf hin, dass sich Unternehmen bei ihren Innovationstätigkeiten häufig eines „local search“ bedienen. Dies bestätigt sich allerdings nur zum Teil in vorliegenden Ergebnissen. Mit Ausnahme von Unternehmen B (Elektroindustrie) und G (Telekommunikation), die beide besonders produktnah ausgerichtet sind, beziehen alle Unternehmen im langen Zeithorizont einen „distant search“ in ihre Prognosen ein, bei dem u.a. auch Entwicklungen in anderen Branchen untersucht werden. Inwiefern die auf diese Weise ermittelten und vom Kerngeschäft entfernter liegenden Prognoseergebnisse auch tatsächlich in die strategische Unternehmens-/Innovationsplanung eingebunden werden, hängt von der bereits gezeigten Nutzung der Prognoseergebnisse ab.

\subsection{Organisatorische Verankerung und prozessuale Ausgestaltung}

Für die organisatorische Verankerung der Zukunftsforschung stehen Unternehmen zahlreiche Optionen zur Verfügung. Die Fallstudien weisen in diesem Zusammenhang den relativ deutlichen Befund aus, dass der Großteil der Unternehmen Foresight-Aktivitäten der zentralen F\&E-Abteilung zuordnet, entweder als eigene Unterabteilung oder als Stabstelle (vgl. Abbildung 6). Hierdurch wird einerseits eine bereichsübergreifende, strategische Ausrichtung des Corporate Foresight ermöglicht, andererseits wird auch die Kopplung der Zukunftsforschung mit den Innovationsaktivitäten der Unternehmen erleichtert. Durch

52 Vgl. hierzu die Hinweise bei Prahalad/Hamel (1990), S. 89, und Helfat/Peteraf(2003), 997ff. 
eine Untergliederung der Zukunftsforschung in bereichsorientierte Teams wird zudem ein Fokus auf den Prognosebedarf einzelner Geschäftsbereiche sichergestellt. Erschwert wird allerdings die Zusammenarbeit mit der Geschäftsführung, was sich u.a. auch darin zeigt, dass bei jenen Unternehmen, die die Zukunftsforschung der F\&E-Abteilung unterordnen, keine regelmäßige Einarbeitung der Prognoseergebnisse in die Unternehmensstrategie erfolgt (vgl. Abbildung 2). Dies kann als erhebliches Defizit in der Praxis der Zukunftsforschung gewertet werden. Überraschend ist, dass lediglich ein Unternehmen das Corporate Foresight als Stabstelle der Geschäftsführung institutionalisiert hat, obgleich durch eine solche organisatorische Verankerung die unmittelbarste Verbindung zur Unternehmensspitze besteht.

\section{Abbildung 2: Organisatorische Verankerung des Corporate Foresight}

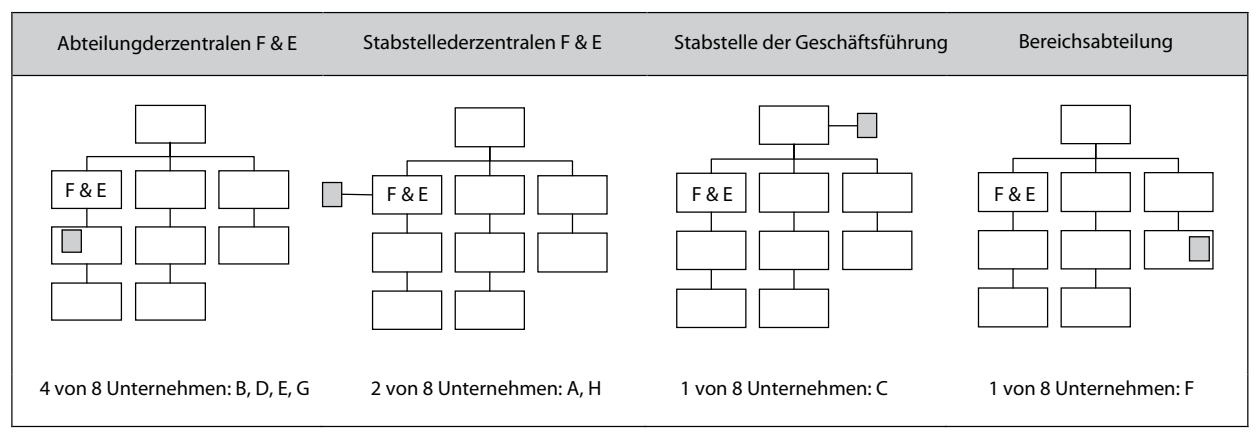

Die ausschließliche Positionierung des Corporate Foresight in den einzelnen Geschäftsbereichen, wie dies ebenso bei einem einzelnen Unternehmen vorliegt, mag zunächst erstaunen. Bei diesem Unternehmen handelt es sich jedoch um einen äußerst diversifizierten Konzern, dessen zentrale F\&E-Abteilung nur eine untergeordnete Rolle einnimmt, so dass sich bislang noch gewisse Vorteile einer dezentralen Ansiedlung der Foresight-Aktivitäten erkennen lassen. Allerdings besteht bei einer solchen Lösung neben dem Nachteil einer kostenintensiven Duplizierung interner Prozesse die oben bereits beschriebene Gefahr, dass strategisch übergreifende, konzernrelevante Fragestellungen von den einzelnen Bereichen vernachlässigt werden.

Obgleich mit der organisatorischen Verankerung des Corporate Foresight bereits zu einem gewissen Maß bestimmt wird, inwiefern die Zukunftsforschung der Unternehmen kontinuierlich oder fallweise stattfinden soll, lassen sich einige markante Unterschiede in den Prozessabläufen erkennen. Wie Abbildung 7 überblicksartig darstellt, realisieren drei Unternehmen das Foresight als kontinuierlichen Prozess. Die Prozesse werden dabei durch spezifische Meilensteine (u.a. Kick-off, Festlegung Innovationsziele, Expertenrunden etc.) und die den eingesetzten Prognosemethoden zugrunde liegende Ablauflogik strukturiert. Zwei der untersuchten Unternehmen führen ihre Aktivitäten der Zukunftsforschung hingegen in Form regelmäßig stattfindender Einzelprozesse durch. Neben der Erstellung von Szenarien werden im Rahmen der Einzelprozesse auch bestimmte Forschungsthemen 
vertieft, die von Bereichsvorständen oder der F\&E-Abteilung vorgeschlagen werden. Die übrigen Unternehmen initiieren einen Foresight-Prozess lediglich fallweise in Form spezifisch definierter Projekte zur Zukunftsforschung. Den Anstoß geben hier z.B. Vorschläge der F\&E-Abteilung, wobei eine konkrete Auftragserteilung zur Durchführung des Foresight-Prozesses i.d.R. erst nach Prüfung durch die Geschäftsführung erfolgt. Regelmäßige Updates zu den einmal erarbeiteten Prognosen werden von diesen Unternehmen nicht durchgeführt. Sie speisen allerdings noch nicht marktreife Ideen ggf. erneut in den Prozess ein und unterziehen sie einer weiteren Evaluation.

Erstaunlich ist, dass von den analysierten Unternehmen kaum die Möglichkeit genutzt wird, Feedbacks zu den erarbeiteten Prognosen und Innovationsideen einzuholen, um damit einen Verbesserungsprozess in der Zukunftsforschung in Gang zu setzen. Feedback-Prozesse zählen zu den Standards modernen Managements und sind zudem auch Bestandteil des klassischen Führungskreislaufs, weshalb es unverständlich ist, dass die Unternehmen hiervon kaum Gebrauch machen. Lediglich die Unternehmen F und $\mathrm{H}$ haben Feedback-Schleifen im Rahmen ihres kontinuierlichen Foresight-Prozesses institutionalisiert.

\section{Abbildung 3: Prozessuale Ausgestaltung des Corporate Foresight}

\begin{tabular}{|l|c|c|c|c|c|c|c|c|}
\hline & \multicolumn{3}{|c|}{ Elektroindustrie } & \multicolumn{2}{c|}{ Automobilindustrie } & Chemie & Telekom. & Halbleiter \\
& A & B & C & D & E & F & G & H \\
\hline $\begin{array}{l}\text { Kontinuierlicher } \\
\text { Prozess }\end{array}$ & & & & $\bullet$ & & & & ・ \\
\hline $\begin{array}{l}\text { Regelmäßige } \\
\text { Einzelprozesse }\end{array}$ & - & & & & & & & \\
\hline $\begin{array}{l}\text { Fallweise } \\
\text { Prozessabläufe }\end{array}$ & & - & $\bullet$ & & - & & & \\
\hline $\begin{array}{l}\text { Einbindung von } \\
\text { Feedback bish. } \\
\text { Forecasts }\end{array}$ & & & & & & & & \\
\hline
\end{tabular}

\subsection{Personelle Ausgestaltung des Corporate Foresight}

Die vorliegenden wissenschaftlichen Erkenntnisse empfehlen eine heterogene Zusammensetzung der mit der Zukunftsforschung betrauten Teams. In der Praxis lässt sich eine entsprechende Teamzusammensetzung bei fast allen Unternehmen beobachten. Neben Interdisziplinarität wird darauf geachtet, spätere Entscheider und Umsetzer so früh wie möglich in den Foresight-Prozess einzubinden (vgl. Abbildung 4). Wie die Fallstudien zeigen, werden oft Vertreter der entsprechenden Fachbereiche in die Foresight-Teams aufgenommen, ebenso wie Personen aus dem Bereich F\&E/Innovationsmanagement. Letztere stellen einen besonders großen Anteil an Mitgliedern der Foresight-Teams in jenen Unternehmen, die die Zukunftsforschung organisatorisch der F\&E-Abteilung zugeordnet haben. Die untersuchten Unternehmen haben die Erfahrung gemacht, dass ausgewiesene Experten im Prozess 
vertreten sein müssen, um die Qualität und Akzeptanz der Prognosen zu steigern. Deshalb greifen die meisten Unternehmen auch auf die Expertise von externen Personen zurück und binden diese lose in die Zukunftsforschung ein (bspw. durch Vorträge).

Bei drei Unternehmen (A, D, F) werden einzelne Personen zu Promotoren ${ }^{53}$ der Zukunftsforschung ernannt. Sie haben die Aufgabe, die Foresight-Aktivitäten sowie damit zusammenhängende Projekte innerhalb des Unternehmens voranzutreiben, dafür notwendige Ressourcen zu akquirieren und (Zwischen-) Ergebnisse intern zu kommunizieren. Zudem vertreten sie die Arbeiten bei Bedarf nach außen. Einen besonderen Fall stellen die Unternehmen B und G dar, die die Zukunftsforschung fallweise in einzelnen Projekten betreiben und diese organisatorisch unter der F\&E-Abteilung verankern. Hier nehmen Projektleiter das Management der nach Bedarf angestoßenen Foresight-Prozesse wahr und können sich ggf. ein eigenes Team für die Analysen zusammenstellen.

Interessant ist die Lösung des Automobilunternehmens D, das wie geschildert verschiedene Innovationsfelder im Rahmen seiner Zukunftsforschung definiert hat. Jedem dieser Felder ist sowohl ein Forschungsleiter als auch ein Promotor zugeordnet, so dass die Zukunftsforschung sowohl fachlich wie unternehmenspolitisch durch dafür bestimmte Personen gesteuert wird. Die einzelnen Projektteams setzen sich vor allem aus Mitarbeitern des Innovationsmanagements zusammen, wobei einzelne Fachbereichsvertreter zu Expertenrunden hinzugezogen werden. Im Vergleich dazu ist die Zusammenstellung der Projektteams beim Automobilhersteller E von Projekt zu Projekt verschieden.

\section{Abbildung 4: Personelle Ausgestaltung des Corporate Foresight}

\begin{tabular}{|l|c|c|c|c|c|c|c|c|}
\hline & \multicolumn{3}{|c|}{ Elektroindustrie } & \multicolumn{2}{|c|}{ Automobilindustrie } & Chemie & Telekom. & Halbleiter \\
& A & B & C & D & E & F & G & H \\
\hline $\begin{array}{l}\text { Einbezug von } \\
\text { Entscheidern / } \\
\text { Umsetzern }\end{array}$ & - & & - & & & & & \\
\hline $\begin{array}{l}\text { (Fallweise) } \\
\text { Partizipation } \\
\text { von ext. } \\
\text { Experten }\end{array}$ & - & - & & & & & & \\
\hline $\begin{array}{l}\text { Vertretung } \\
\text { nach innen } \\
\text { und außen }\end{array}$ & Promotoren & Projektleiter & & Promotoren & & Promotoren & Projektleiter & \\
\hline $\begin{array}{l}\text { Management- } \\
\text { unterstützung }\end{array}$ & stark & fallweise & stark & stark & fallweise & schwach & fallweise & stark \\
\hline
\end{tabular}

53 Vgl. hierzu die Arbeiten von Witte (1973), der den Begriff „Promotor“ in die Innovationsliteratur eingeführt hat, und von Hauschildt/Gemünden (1999). 


\section{Diskussion der Ergebnisse, Empfehlungen und Ausblick}

Die vorliegende Untersuchung weicht deutlich von der Bahn der bisherigen Arbeiten zur Zukunftsforschung von Unternehmen ab, indem sie den Fokus nicht auf die Prognoseverfahren legt, sondern weitere zentrale Aspekte des Managements der Zukunftsforschung berücksichtigt. Wie gezeigt werden konnte, existiert ein relativ großer Gestaltungsspielraum hinsichtlich inhaltlicher, organisatorischer, prozessualer und personeller Aspekte. Dieser Spielraum wird jedoch nur von wenigen Unternehmen und nicht in allen Aspekten genutzt.

Für das Management des Corporate Foresight ist zunächst die Erkenntnis wegleitend, dass sich die langfristige Zukunftsforschung grundlegend von der Erstellung kurzfristiger Prognosen, wie sie in jedem Geschäftsbereich erfolgt, unterscheidet. Neben der offensichtlichen Wahl geeigneter Verfahren für die langfristige Prognose ist deshalb mit Blick auf die Qualität, Akzeptanz und Umsetzung der Prognoseergebnisse auf weitere Gestaltungsdimensionen zu achten.

Ein Vergleich der Unternehmen lässt erkennen, dass manche Gestaltungsdimensionen des Corporate Foresight recht homogen, manche aber auch recht heterogen realisiert werden. So sind vor allem bei der Wahl der Prognoseverfahren kaum Unterschiede zwischen den Unternehmen auszumachen. Die Unternehmen greifen auf ein ähnliches Set an Verfahren zurück, wobei die Szenarioanalyse das wichtigste Verfahren ist. Die relativ universelle Anwendbarkeit dieses Verfahrens kann seine weite Verbreitung zum Teil erklären, ebenso wie seine Bekanntheit im Fachbereich Innovationsmanagement.

Hingegen lassen sich bei den weiteren Gestaltungsdimensionen mithin sehr deutliche Unterschiede zwischen den Unternehmen erkennen. Diese auch innerhalb einzelner Branchen beobachtbare Heterogenität kann zum Teil darauf zurückgeführt werden, dass ein Benchmarking zum Corporate Foresight aufgrund der meist strikten Geheimhaltung von Informationen zur Zukunftsforschung schwer fällt. Ein weiterer Grund ist, dass auch in der Literatur erst ansatzweise Wissen zu den diskutierten Gestaltungsdimensionen vorhanden ist, wohingegen die Prognoseverfahren gut dokumentiert sind.

Wie die Analyse der Fallstudien ergeben hat, nutzt der Großteil der Unternehmen die Zukunftsforschung um mögliche Trends und Szenarien zu erkennen und konkrete Handlungsempfehlungen für einzelne Bereiche abzuleiten. Eine regelmäßige Einarbeitung der Ergebnisse in die Unternehmensstrategie erfolgt jedoch erst bei wenigen Unternehmen. Zudem fällt auf, dass nur wenige Unternehmen die Möglichkeit nutzen, formelles Feedback zu den erarbeiteten Prognosen bzw. Innovationsideen von den Geschäftsbereichen bzw. nachgelagerten F\&E-Abteilungen einzuholen. Die Mehrzahl der Unternehmen verlässt sich hier auf informelle Kommunikationskanäle, was u.U. dazu führt, dass negatives Feedback nicht weitergeleitet wird. Bei jenen Unternehmen, die Projekte der Zukunftsforschung nur fallweise betreiben und wechselnde Mitarbeiter damit betrauen, besteht zudem die Gefahr, dass die gesammelten Erfahrungen nicht zur weiteren Verbesserung der Zukunftsforschung genutzt werden. 
Wie die Unternehmensvertreter betont haben, ist bei der Durchführung der Zukunftsforschung auf die Akzeptanz der Prognoseergebnisse in den betroffenen Bereichen zu achten. Die Geheimhaltung der Foresight-Projekte kann leicht zu einer Verunsicherung für betroffene, jedoch nicht beteiligte Bereiche führen und den Willen zur späteren Umsetzung beeinträchtigen. Der Großteil der untersuchten Unternehmen legt deshalb einen besonderen Wert darauf, spätere Entscheider und Umsetzer möglichst früh einzubinden. Die Unternehmen bewegen sich dabei allerdings in einem Spannungsfeld zwischen Preisgabe von strategisch wichtigen Informationen und deren Geheimhaltung.

Die praktischen Erfahrungen der Unternehmen haben ebenso gezeigt, dass bei der Zukunftsforschung besonderes Augenmerk auf die Motivation der beteiligten Personen zu legen ist. So läuft das Tagesgeschäft für die Mitarbeiter nahezu aller Unternehmen in unveränderter Intensität weiter, obgleich mit der Durchführung von Szenarioanalysen und anderen Prognoseverfahren ein hoher zeitlicher Aufwand verbunden ist. Wie den Expertengesprächen zu entnehmen war, wirkt sich die entstandene Zusatzbelastung nicht selten demotivierend auf die beteiligten Mitarbeiter aus. Motivierend wirkt hingegen das für die Zukunftsforschung aufgebrachte Interesse des obersten Managements, weil es den Stellenwert der geleisteten Arbeit für das Gesamtunternehmen verdeutlicht.

Erstaunlich ist, dass alle Unternehmen in ihren Aktivitäten der Zukunftsforschung nach dem Aufbau neuer organisationaler Handlungsfelder suchen, die Aktivitäten jedoch nicht bzw. nur indirekt dazu nutzen, um den Abbau bestimmter Aktivitäten bzw. den Rückzug aus bestimmten Geschäftsfeldern zu untersuchen. Das mit dem Corporate Foresight verbundene Erkenntnispotenzial zur evolutorischen Gestaltung von Unternehmen wird daher nur zum Teil ausgeschöpft.

Zusammenfassend zeigt Abbildung 5, dass unabhängig von der Branche und in Ergänzung des bisherigen, rein methodenorientierten Verständnisses verschiedene Gestaltungsaspekte beim Management der Zukunftsforschung zu berücksichtigen sind. Dabei deuten sich aus der Gesamtschau der Fallstudien drei typische Muster der Ausgestaltung der Zukunftsforschung an, die drei unterschiedlichen Ansätzen zum Management dieses Bereichs entsprechen und deshalb als erste Leitlinien für die Konzeption der Zukunftsforschung dienen können. Zur Vermeidung einer aufwendigen Duplizierung und zur Professionalisierung der Zukunftsforschung empfiehlt sich meist nur die Realisierung in Form einer Stabstelle der Geschäftsführung oder bedingt auch in Form einer Stabstelle/Abteilung der zentralen F\&E (Ansätze I und II in Abbildung 5).

Zu berücksichtigen ist, dass die jeweilige organisatorische Verankerung der Zukunftsforschung deren inhaltliche Ausrichtung, wie in Abbildung 9 dargestellt, maßgeblich prädisponiert. So liegt bspw. einer Stabstelle der Geschäftsführung eine Beschäftigung mit der konzernweiten Unternehmensstrategie näher als einer Stabstelle/Abteilung der zentralen F\&E. Umgekehrt befasst sich eine Stabstelle/Abteilung der zentralen F\&E eher mit Fragestellungen der künftigen Technologieentwicklung als eine Stabstelle der Geschäftsführung.

Für das Management der Zukunftsforschung ist es also wichtig, sich diese grundsätzlichen Zusammenhänge bewusst zu machen und die gewünschte inhaltliche Ausrichtung des 
Corporate Foresight auf die Unternehmensentwicklung mit gezielten Maßnahmen (z.B. Zusammensetzung des Foresight-Teams) herbeizuführen. Wie das Fallstudienunternehmen F illustriert, kann eine solche Ausrichtung auf die künftige Unternehmensentwicklung auch bei Verfolgung von Ansatz III gelingen. Allerdings sind hiermit die bereits diskutierten Schwierigkeiten verbunden.

\section{Abbildung 5: Drei typische Ansätze der Zukunftsforschung}

\begin{tabular}{|c|c|c|c|}
\hline $\begin{array}{l}\text { Organisatorische } \\
\text { Verankerung }\end{array}$ & $\begin{array}{c}\text { I. } \\
\text { Stabstelle der } \\
\text { Geschäftsführung }\end{array}$ & $\begin{array}{c}\text { Il. } \\
\text { Stabstelle/Abteilung der } \\
\text { zentralen F\&E }\end{array}$ & $\begin{array}{c}\text { III. } \\
\text { Abteilung auf } \\
\text { Geschäftsbereichsebene }\end{array}$ \\
\hline & $\begin{array}{l}\text { v.a. unternehmensweite, } \\
\text { strategische Fragestellungen }\end{array}$ & $\begin{array}{l}\text { v.a. technologieorientierte } \\
\text { Fragestellungen }\end{array}$ & $\begin{array}{l}\text { v.a. markt-/produktnahe } \\
\text { Fragstellungen }\end{array}$ \\
\hline $\begin{array}{l}\text { Inhaltliche } \\
\text { Ausgestaltung } \\
\text { (Prädisposition) }\end{array}$ & $\begin{array}{l}\text { Zeithorizont: } \\
\text { bis } 20 \text { Jahre }\end{array}$ & $\begin{array}{l}\text { Zeithorizont: } \\
\text { bis } 20 \text { Jahre } \\
\text { Ableitung von }\end{array}$ & $\begin{array}{l}\text { Zeithorizont: } \\
\text { bis } 5 \text { Jahre }\end{array}$ \\
\hline & $\begin{array}{l}\text { Einbindung der Ergebnisse in } \\
\text { die Unternehmensstrategie }\end{array}$ & $\begin{array}{l}\text { Handlungsempfehlungen } \\
\text { (evtl. ohne konsequente } \\
\text { Strategieeinbindung) }\end{array}$ & $\begin{array}{l}\text { Eigene Umsetzung } \\
\text { der Ergebnisse in den } \\
\text { Bereichen }\end{array}$ \\
\hline Prozessuale & \multicolumn{3}{|c|}{ Einbindung aller Aktivitäten in einen übergeordneten, kontinuierlichen Gesamtprozess } \\
\hline Ausgestaltung & \multicolumn{3}{|c|}{ Feedbackschleifen im Team und zu den „Umsetzern“ } \\
\hline Personelle & \multicolumn{3}{|c|}{ Einbindung aller relevanten, von den Prognosen betroffenen Abteilungen } \\
\hline Ausgestaltung & \multicolumn{3}{|c|}{ Sicherstellung einer angemessenen Unterstützung durch die Geschäftsführung } \\
\hline
\end{tabular}

Als Gesamtfazit bleibt festzuhalten, dass es sich bei der Zukunftsforschung von Unternehmen um ein bislang nur einseitig erforschtes Gebiet handelt. Für Forscher, die sich dieses Bereichs annehmen wollen, ergeben sich damit zahlreiche Möglichkeiten, sowohl theoretisch bedeutsamen als auch praxisrelevanten Forschungsfragen nachzugehen.

\section{Literatur}

Ansoff, H. Igor (1976), Managing Surprise and Discontinuitiy - Strategic Response to Weak Signals, in: zfbf, 8. Jg, S. 129-152.

Armstrong, J. Scott (1986), The Ombudsman: Research on Forecasting: A Quarter-Century Review, 1960-1984, in: Interfaces, Vol. 16, Nr. 1, S. 89-109.

Barney, Jay (1991), Firm Resources and Sustained Competitive Advantage, in: Journal of Management, Vol. 17, No. 1, S. 99-120.

Bonner, Joseph. M./Ruekert, Robert. W./ Walker, Orville. C. (2002), Upper Management Control of New Product Development Projects and Project Performance, in: Journal of Product Innovation Management, Vol. 19, Nr. 3, S. 233-245.

Bright, James R. (1968), Technological Forecasting for Industry and Government: Methods and Applications. Brockhoff, Klaus (1977), Prognoseverfahren für die Unternehmensplanung. 
Bürgel, Hans Dietmar/Reger, Guido/Ackel-Zakour, Rene (2002), Technologie-Früherkennung in multinationalen Unternehmen: Ergebnisse einer empirischen Untersuchung, in: Möhrle, Martin G.IIsenmann, Ralf (Hrsg.): Technologie-Roadmapping - Zukunftsstrategien für Technologieunternehmen, S. 19-46.

Burgelman, Robert A./Maidique, Modesto A. (1988): Strategic Management of Technology and Innovation.

Cetron, Marvin J. (1971): Technological Forecasting - A practical Approach.

Coates, Jospeh F (1985), Foresight in Federal Government Policy making, in: Futures Research Quarterly, Vol. 1, Summer Issue, S. 29-53.

Cohen, Wesley M./Levinthal, Daniel A. (1990), Absorptive Capacity: A New Perspective on Learning and Innovation, in: Administrative Science Quarterly, Vol. 35, S. 128-152.

Conway, H. Allan/McGuiness, Norman W. (1986), Idea generation in technology-based firms, in: Journal of Product Innovation Management, Vol. 3, Nr. 4, S. 276-291.

Cooper, Lee G. (2000), Strategic Marketing Planning for Radically New Products, in: Journal of Marketing, Vol. 64, January, S. 1-16.

Cuhls, Kerstin (2003), From Forecasting to Foresight Processes - New Participative Foresight Activities in Germany, in: Journal of Forecasting, Vol. 22, S. 93-111.

Daniel, D. Ronald (1961), Management Information Crisis, in: Harvard Business Review, Vol. 39, Sept.-Oct., S. 111-121.

Day, George S. (1994a), Continuous Learning about Markets, in: California Management Review, Vol. 36, No. 3, S. 9-31.

Day, George S. (1994b), The Capabilities of Market-Driven Organizations, in: Journal of Marketing, Vol. 58, October, S. 37-52.

Day, George S./Nedungadi, Prakash (1994), Managerial Representations of Competitive Positioning, in: Journal of Marketing, Vol. 58, April, S. 31-44.

Day, George S./Schoemaker, Paul J.H. (2000), Avoiding the Pitfalls of Emerging Technologies, in: California Management Review, Vol. 42, No. 2, S. 8-33.

Dierickx, Ingemar/Cool, Karel (1989), Asset Stock Accumulation and Sustainability of Competitive Advantage, in: Management Science, Vol. 35, No. 12, S. 1504-1511.

Dougherty, Deborah (2002), Grounded Theory Research Methods, in: Baum, J. A. C. (Hrsg.): The Blackwell Companion to Organizations, S. 849-866.

Eisenhardt, Katbleen M. (1989), Building Theories from Case Study Research, in: Academy of Management Review, Vol. 14, No. 4, S. 532-550.

Elikai, Fara/Hall Jr., William/Elikai, Phyllis (1999), Managing and Improving the Forecasting Process, in: The Journal of Business Forecasting, Vol.18, No. 1, S. 15-19.

Ernst, Holger (2003), Ursachen eines Informant Bias und dessen Auswirkung auf die Validität empirischer betriebswirtschaftlicher Forschung, in: ZfB, 73. Jg., Nr. 12, S. 1249-1275.

Folkerts, Liesa/Hauschildt, Jürgen (2002), Personelle Dynamik in Innovationsprozessen, in: Die Betriebswirtschaft, 62. Jg, Nr. 1, S. 7-23.

Gausemeier, Jürgen/Fink, Alexander/Schlake, Oliver (1998), Scenario Management: An Approach to Develop Future Potentials, in: Technological Forecasting and Social Change, Vol. 59, S. 111-130.

Gavetti, Giovanni/Levinthal, Daniel (2000), Looking Forward and Looking Backward: Cognitive and Experential Search, in: Administrative Science Quarterly, Vol. 45, March, S. 113-137.

Geschka, Horst/Hammer, Richard (1997), Die Szenario-Technik in der strategischen Unternehmensplanung, in: Hahn, Dietger/Taylor, Bernard (Hrsg.): Strategische Unternehmensplanung - Strategische Unternehmensführung: Stand und Entwicklungstendenzen, S. 465-489.

Glaser, Barney G./Strauss, Anselm L. (1967), The Discovery of Grounded Theory: Strategies for Qualitative Research. 


\section{GRUBER/C. VENTER}

Godet, Michel (2000), The Art of Scenarios and Strategic Planning: Tools and Pitfalls, in: Technological Forecasting and Social Change, Vol. 65, S. 3-22.

Gruber, Marc/Kolpatzik, Bernd/Schönhut, Jürgen/Venter, Claudia (2003), Die Rolle des Corporate Foresight im Innovationsprozess: Ziele, Ausgestaltung und Erfahrungen am Beispiel der Siemens AG, in: Zeitschrift Führung + Organisation (zfo), Nr. 5, S. 285-290.

Grupp, HariolflLinstone, Harold A. (1999), National Technology Foresight Activities around the Globe, in: Technological Forecasting and Social Change, Vol. 60, S. 85-94.

Hamel, Gary/Prahalad, C.K. (1994): Competing for the Future.

Hardie, Bruce G. S./Fader, Peter S./Wisniewski, Michael (1998), An Empirical Comparison of new Product Trial Forecasting Models, in: Journal of Forecasting, Vol. 17, Nr. 3-4, S. 259-280.

Hauschildt, Jürgen/Gemünden, Hans Georg (1999), Promotoren - Champions der Innovation.

Helfat, Constance E. (2000), Guest Editor's Introduction to the Special Issue: The Evolution of Firm Capabilities, in: Strategic Management Journal, Vol. 21, S. 955-960.

Helfat, Constance E./Peteraf, Margaret A. (2003), The Dynamic Resource-Based View: Capability Lifecycles, in: Strategic Management Journal, Vol. 24, S. 997-1010.

Hogarth, Robin M./Makridakis, Spyros (1981), Forecasting and Planning: An Evaluation, in: Management Science, Vol. 27, Nr. 2, S. 115-138.

Jain, Chaman L. (2002a), Explosion in the Forecasting Function, in: The Journal of Business Forecasting, Vol. 21, Fall, S. 2-4.

Jain, Chaman L. (2002b), Benchmarking the Background of Business Forecasters, in: The Journal of Business Forecasting, Vol. 21, Fall, S. 28.

Jain, Chaman L. (1999), Explosion in the Forecasting Function in Corporate America, in: The Journal of Business Forecasting, Vol. 18, Summer, S. 2 u. 28.

Kahn, Kenneth B. (2002), An exploratory Investigation of new Product Forecasting Practices, in: Journal of Product Innovation Management, Vol. 19, S. 133-143.

Kappel, Thomas A. (2001), Perspectives on Roadmaps: How Organizations talk about the Future, in: Journal of Product Innovation Management, Vol. 18, S. 39-50.

Kress George J./Snyder John (1994), Forecasting and Market Analysis Techniques: A Practical Approach.

Lapide, Larry (1997), New Developments in Business Forecasting, in: The Journal of Business Forecasting, Vol. 16, Winter, S. 32.

Lamnek, Siegfried (1995), Qualitative Sozialforschung, Band 1, Methodologie.

Lawless, Mark J. (1997), Forecasting in the 1990's, in: The Journal of Business Forecasting, Vol. 16 (1997), Fall, S. 9-12.

Leonard-Barton, Dorothy (1992), Core Capabilities and Core Rigidities: A Paradox in Managing New Product Development, in: Strategic Management Journal, Summer Special issue, Vol. 13, S. 111-126.

Levinthal, Daniel A./March James G. (1993), The Myopia of Learning, in: Strategic Management Journal, Vol. 14, S. 95-112.

Levitt, Barbara/March, James G. (1993), Organizational Learning, in: Annual Review of Sociology, Vol. 14, S. 319-340.

Liebl, Franz (1996), Strategische Frühaufklärung: Trends - Issues - Stakeholders.

Mackay, Marisa Maio/Metcalfe, Mike (2002), Multiple Method Forecasts for Discontinuous Innovations, in: Technological Forecasting and Social Change, Vol. 69, S. 221-232.

March, James G. (1994), Exploration and Exploitation in Organizational Learning, in: Organization Science, Vol. 2, No. 1, S. 71-87.

Martin, Ben R. (1999), Technology Foresight in a Rapidly Globalizing Economy, in: SPRU - Science and Technology Policy Research, University of Sussex 1999.

Martin, Xavier/Mitchell, Will (1998), The Influence of Local Search and Performance Heuristics on New Design Introduction in a New Product Market, in: Research Policy, Vol. 26, No. 7/8, S. 753-771. 
Mayring, Philipp (1990), Einführung in die qualitative Sozialforschung.

McGrath, Rita Gunther (1997), Falling Forward: Real Options Reasoning and Entrepreneurial Failure, in: Academy of Management Review, Vol. 24, No. 1, S. 13-30.

Müller-Stewens, Günter (1990), Strategische Suchfeldanalyse (2. Aufl.).

Porter, Alan L./Roper, A. Thomas/Mason, Thomas W./Rossini, Frederick A./Banks, Jerry (1991), Forecasting and Management of Technology.

Prahalad, C.K./Hamel, Gary (1990), The Core Competence of the Corporation, in: Harvard Business Review, Vol. 68, May/June, S. 79-91.

Prehoda, Robert W. (1967), Designing the Future - the Role of Technological Forecasting, London, in: Bright, James R. (Hrsg.): Technological Forecasting for Industry and Government: Methods and Applications.

Reibnitz, Ute v. (1992), Szenario-Technik - Instrumente für die unternehmerische und persönliche Erfolgsplanung (2. Aufl.).

Roberts, Edward B. (1995), Benchmarking the Strategic Management of Technology - I, in: Research and Technology Management, Vol. 38, January/February, S. 44-56.

Rosenkopf, Lori/Almeida, Paul (2001), Overcoming Local Search through Alliances and Mobility. Working Paper.

Rosenkopf, Lori/Nerkar, Atul (2001), Beyond Local Search: Boundary-spanning, Exploration, and Impact in the Optical Disk Industry, in: Strategic Management Journal, Vol. 22, S. 287-306.

Salo, Ahti/Gustafsson, Tommi/Ramanathan, Ramakrishnan (2003), Multicriteria Methods for Technology Foresight, in: Journal of Forecasting, Vol. 22, S. 235-255.

Sorenson, Jesper B./Stuart, Toby E. (2000), Aging, Obsolescence and Organizational Innovation, in: Administrative Science Quarterly, Vol. 45, S. 81-112.

Stier, Winfried (1999), Empirische Forschungsmethoden (2. Aufl.).

Strauss, Anselm/Corbin, Juliette (1990), Basics of Qualitative Research: Grounded Theory Procedures and Techniques.

Stuart, Toby E./Podolny, Joel M. (1996), Local Search and the Evolution of Technological Capabilities, in: Strategic Management Journal, Summer Special issue, Vol. 17, S. 21-38.

Staw, Barry M./Ross, Jerry (1987), Behaviours in Escalation Situations: Antecedents, Prototypes, and Solutions, in: Cummings, Larry G./Staw, Barry. (Hrsg.): Research in Organizational Behaviour, S. 39-78.

Teece, David J./Pisano, Gary/Shuen, Amy (1997), Dynamic Capabilities and Strategic Management, in: Strategic Management Journal, Vol. 18, S. 509-533.

Trigeorgis, Lenos (1999), Real Options: Managerial Flexibility and Strategy in Resource Allocation.

Vanston, John H. (1995), Technology Forecasting: An Aid to effective Technology Management.

Veryzer, Richard W. (1998), Discontinuous Innovation and the New Product Development Process, in: Journal of Product Innovation Management, Vol. 15, S. 304-321.

Weyrich, Claus (2002), Zukunft erfinden - Strategisches Innovationsmanagement (Teil II), in: Frankfurter Allgemeine Zeitung, Nr. 28 vom 2.2.2002, S. 73.

Williams, Katherine Y./O'Reilly, Charles A. (1998): Demography and Diversity in Organizations: A Review of 40 Years of Research, in: Research on Organizational Behavior, Vol. 20, S. 77-140.

Wilson, Ian (2000), From Scenario Thinking to Strategic Action, in: Technological Forecasting and Social Change, Vol. 65, S. 23-29.

Witte, Eberhard (1973), Organisation von Innovationsentscheidungen: Das Promotoren-Modell.

Wolfrum, Bernd (1995), Strategisches Technologiemanagement.

Yin, Robert K. (1994): Case Study Research: Design and Methods. 


\section{Summary}

Studies in management have acknowledged the importance of corporate foresight activities since the 1960 ies. Yet, research in this area has dealt almost exclusively with various techniques of forecasting, thus neglecting several major issues concerning the management of corporate foresight activities, e.g. the introduction of a foresight function, the selection of personnel for foresight activities and the specific content of forecasts.

Following a grounded theory approach, the purpose of this study is to explore the practice of foresight and to generate stimulating insights into this important, yet not well-understood area. Comprehensive case-based data was collected from eight major German corporations.

Our results indicate that firms do not make full use of the possibilities corporate foresight has to offer, thus leaving room for further improvement. Furthermore, our findings suggest three typical approaches to corporate foresight. Results are discussed with reference to recent studies in innovation management and in strategic management. 